\title{
EchoGéo
}

$13 \mid 2010$

Afrique, 50 ans d'indépendance : État et territoires

\section{L'aménagement du territoire tunisien : 50 ans de politiques à l'épreuve de la mondialisation}

\section{Najem Dhaher}

\section{(2) OpenEdition}

1 Journals

\section{Édition électronique}

URL : https://journals.openedition.org/echogeo/12055

DOI : $10.4000 /$ echogeo. 12055

ISSN : 1963-1197

\section{Éditeur}

Pôle de recherche pour l'organisation et la diffusion de l'information géographique (CNRS UMR 8586)

Référence électronique

Najem Dhaher, «L'aménagement du territoire tunisien : 50 ans de politiques à l'épreuve de la mondialisation », EchoGéo [En ligne], 13 | 2010, mis en ligne le 20 septembre 2010, consulté le 03 août 2021. URL : http://journals.openedition.org/echogeo/12055; DOI : https://doi.org/10.4000/echogeo. 12055

\section{Ce document a été généré automatiquement le 3 août 2021}

EchoGéo est mis à disposition selon les termes de la licence Creative Commons Attribution - Pas d'Utilisation Commerciale - Pas de Modification 4.0 International (CC BY-NC-ND) 


\title{
L'aménagement du territoire tunisien : 50 ans de politiques à l'épreuve de la mondialisation
}

\author{
Najem Dhaher
}

\section{Introduction}

1 Depuis son indépendance en 1956, la Tunisie a connu de profondes transformations qui ont rendu indispensable la redéfinition de la politique d'aménagement du territoire. Cette dernière a suivi globalement les grandes étapes que le pays a connues. La planification économique a toujours devancé l'aménagement du territoire, ce dernier se manifestant progressivement dans les années 1960, sans être vraiment établi sous forme d'un programme planifié. Durant les trente premières années de l'indépendance, l'aménagement du territoire s'est inscrit dans une approche sectorielle de l'action de l'Etat et d'une planification économique plutôt verticale. Les disparités entre les régions héritées de la période coloniale se perpétuent voire s'amplifient. En effet, les espaces à l'écart des dynamiques métropolitaines connaissent un affaissement de leur tissu productif et dépendent de plus en plus des emplois publics. Il a fallu attendre 1985 pour voir la naissance du premier schéma national d'aménagement du territoire, et des schémas régionaux. Le changement politique de $1987^{1}$ a eu des répercussions sur les politiques nationales de développement. Ces dernières se traduisent, au début des années 1990, par une réflexion sur l'aménagement du territoire via des investissements économiques, l'équipement et la promotion administrative des villes moyennes. Les transformations politiques et institutionnelles liées à l'augmentation de l'aide internationale ont introduit des changements significatifs de l'architecture administrative $^{2}$. Actuellement, le développement des villes tunisiennes, et particulièrement celui des plus dynamiques d'entre elles, est en train de se concevoir dans le cadre des enjeux nés de la globalisation. Les documents officiels (journal officiel de la république tunisienne, plan de développement économique et social) et les schémas d'aménagement actuels, notamment le nouveau schéma directeur 
d'aménagement du territoire, semblent opter pour des choix renforçant des tendances de développement sélectif et différencié. Cette orientation d'ouverture et d'insertion dans le «mondialibéralisme" a impliqué une politique de réforme et de restructuration dite de "mise à niveau intégrale». D'ailleurs, le Ministère de l'Equipement et de l'Habitat (MEH-SIDES-URBAPLAN, 1996) reconnaît que «les politiques et actions urbaines doivent faire une large part à ce qui est reconnu comme étant les facteurs de compétitivité économique ».Comment alors les politiques territoriales et urbaines qui essayent d'instaurer une organisation spatiale et territoriale équilibrée et conforme aux enjeux du développement durable ont-elles été construites pour intégrer les contraintes de la mondialisation?

2 On analysera d'abord les grandes étapes de l'aménagement du territoire depuis l'indépendance, pour aborder ensuite les enjeux de gouvernance territoriale associée à la phase actuelle de mondialisation.

\section{La construction nationale et l'aménagement du territoire}

\section{Difficultés de traitement des disparités spatiales (1956-1969)}

3 Après l'indépendance, les pouvoirs publics tunisiens se sont trouvés devant l'obligation de gérer le contraste entre les différentes régions. La construction nationale a fortement orienté les politiques d'aménagement spatial conduites par l'Etat. Lepremier document de planification économique et sociale, appelé Perspectives décennales de développement, a été élaboré en 1961. Ce document a souligné les fortes disparités régionales, tant au niveau de la répartition des activités que de la population. L'orientation politique qui vise principalement la «tunisification» de l'appareil de production durant cette période oscille entre les impératifs de la construction nationale, tant à l'échelle nationale qu'à celui de la métropole Tunis, et la nécessité de faire face aussi bien aux déséquilibres et aux dysfonctionnements territoriaux qu'à la course à l'urbanisation, qui obligent de plus en plus l'Etat à équiper et à aménager les villes.

4 Cet impératif a amené l'Etat à procéder à un découpage spatial qui réponde aux enjeux de la modernisation. Ainsi, un nouvel échelon territorial intermédiaire a été institué entre le gouvernorat et les petits secteurs appelés jusque là Imadas. Entre 1956 et 1959, le nombre de communes est passé de 75 à 112. Ce découpage administratif a permis, d'après une étude de Belhédi, de dépasser les cadres traditionnels des tribus ${ }^{3}$ et de renforcer la cohésion sociale (Belhédi, 1992). Cependant, la multiplication des collectivités locales et l'amélioration des services administratifs et d'équipement n'ont pas contribué à réduire sensiblement les disparités régionales. En effet, les péripéties qui ont accompagné ce premier découpage du territoire en disent long: certains dirigeants du pays ont tenu à faire de leurs villes des chefs-lieux de régions administratives (comme le premier président Bourguiba à Monastir). Ces logiques ont produit des découpages spatiaux inégalitaires, soulignés par l'importance relative d'un milieu rural défavorisé et peu peuplé.

5 Avec 51\% de la population totale du pays en 1956, la frange littorale consommait à cette époque $89 \%$ de la production de l'électricité. Elle concentrait la quasi-totalité de la production industrielle, $84 \%$ des lits d'hôpitaux, $84 \%$ des médecins et $70 \%$ des élèves des 
écoles primaires selon le recensement général de la population et de l'habitat publié en 1966. Ces disparités caractérisaient déjà le paysage tunisien à la veille de l'indépendance. Les arrangements constitutifs $\mathrm{du}$ régime politique issu de l'indépendance, centralisé et imprégné sur le plan technique des logiques modernistes, commencent à se manifester. Les politiques de développement humain n'arrivent pas à réduire les écarts, à cause des dynamiques et des inerties régionales. Les variations dans la répartition spatiale de la population s'expliquent à la lumière d'un héritage territorial colonial : fonctionnement extraverti du système urbain, macrocéphalie de la capitale, prépondérance des villes ports, sous-équipement de certaines zones demeurées en marge, etc. (Signoles, 1985).

6 L'aménagement, jusque là rattaché à la planification économique, s'autonomise en 1961 avec la création d'un service de l'aménagement du territoire et de l'urbanisme au sein du secrétariat aux travaux publics et à l'habitat. Ce changement a permis une relative ouverture sur la Tunisie intérieure. L'effort entrepris dans la période dite d'« économie planifiée » (1962-1969) a permis d'élargir sensiblement l'espace industriel en implantant des usines dans les régions laissées jusqu'alors totalement à l'écart (Kasserine, Kef, Béja, El Ksour, ElHamma). Si les interventions en faveur d'une organisation spatiale des agents et des services ont permis à cette politique d'aménagement du territoire "par le haut » de répartir les emplois sur beaucoup de régions défavorisées, elles n'ont pas pu atteindre un certain équilibre spatial (Belhédi, 1990). En fait, au cours de cette période, la caractéristique principale résidait dans la consolidation de l'armature urbaine, presque exclusivement effectuée à l'initiative de l'Etat. Mais, compte tenu des héritages à assumer et de certains choix d'investissement, c'est davantage le tertiaire public que l'industrie qui a été le moteur de l'urbanisation et le moyen privilégié de réorganisation du système spatial. En 1969, un ministère de l'aménagement $d u$ territoire et $d u$ tourisme a été institué. L'association entre aménagement du territoire et tourisme traduit l'essor fulgurant de ce secteur et la nécessité d'un aménagement spatial sectoriel spécifique.

\section{Le tournant libéral et la transformation de l'organisation spatiale (1970-1986)}

7 Au début des années 1970, une stratégie d'industrialisation tirée par l'exportation remplace celle qui s'appuyait sur le marché intérieur. Ce changement du moteur principal de croissance a nécessité la mise en place du Code de l'investissement en 1969. La loi d'avril 1972 et celle d'août 1974 traduisent aussi l'option libérale et l'ouverture au capital privé sous forme d'investissement direct. Les migrations rurales des années 1970 expriment la libéralisation économique et le résultat d'une conjonction de facteurs : étatisation des domaines coloniaux, privatisation des terres, forte croissance démographique. Tout cela, dans un contexte de réformes agraires successives, après la brutale expérience collectiviste ${ }^{4}$ des coopératives agricoles, qui a précipité cette ouverture économique.L'essor de l'industrie, le développement du tourisme, le mouvement spontané d'urbanisation consécutif à l'occupation irrégulière des terres publiques et privées, ont créé l'essentiel des villes (Chabbi, 1998). Cette situation a rendu nécessaire l'élaboration d'un cadre de référence, traduisant l'intérêt des pouvoirs publics pour l'aménagement du territoire. Dans cette optique, la direction de l'aménagement du territoire lança en 1975 une étude sur la décentralisation industrielle. En 1979, une commission ad hoc a été chargée de réfléchir aux problèmes 
de l'aménagement. Pourtant, c'est un texte de $1943^{5}$ qui a servi de base à l'aménagement de l'espace urbain jusqu'à la fin des années 1970. D'ailleurs, le Code de l'urbanisme de 1979 n'en serait en réalité qu'une simple révision (Belhédi, 1990). Il est vrai que ce code est resté muet quant à l'articulation entre urbanisme, aménagement du territoire et planification socio-économique. La multiplication des structures (AFH, ARRU, AUGT, APAL, PDR, etc. $)^{6}$ sans coordination a conduit à des politiques incohérentes. Le mimétisme par rapport à la période coloniale se retrouve aussi dans les textes réglementaires et dans les institutions concernées par l'aménagement du territoire ${ }^{7}$. Bugnicourt (1978) évoque à ce sujet l'« idéologie mimétique » à propos de l'invocation des métropoles d'équilibre et de la régionalisation. Certes, l'affinage du découpage administratif depuis 1974 a permis à de nombreux centres de bénéficier de services régionaux ou locaux ${ }^{8}$. Cependant, malgré l'effort entrepris entre 1970 et 1980 par le desserrement de l'encadrement administratif et par l'organisation des services sanitaires, les disparités régionales persistent (Miossec; Signoles, 1984). Depuis, on assiste à un passage progressif d'une littoralisation plutôt passive à une littoralisation active (Béthemont, 2001).

Illustration 1 - Evolution de l'urbanisation en Tunisie

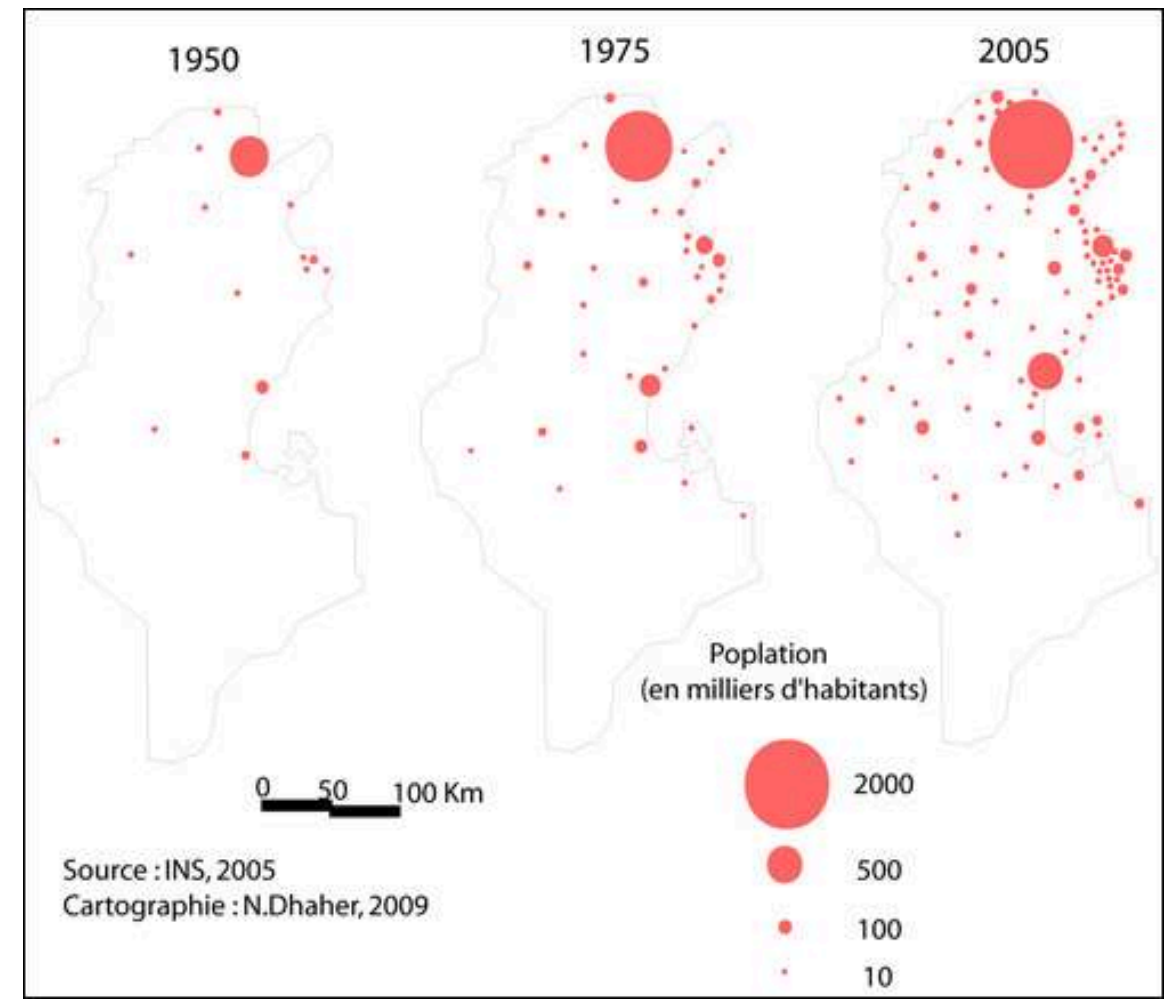

Source : INS, 2005.

Cette logique d'organisation de l'espace a créé de nouveaux rapports entre l'armature urbaine et le monde rural et relativisé la place de l'agriculture dans le développement des espaces ruraux. La Tunisie intérieure est demeurée à l'écart des investissements privés tout en bénéficiant d'un mince effort de l'Etat. Au milieu des années 1980, la crise économique et politique (crise de la dette, suppression des subventions aux produits de première nécessité qui provoquent « la révolte du pain » en janvier 1984) amène l'Etat à opter pour une plus grande ouverture. Cette réalité pousse les pouvoirs 
publics à établir en 1985 le premier schéma national d'aménagement du territoire. Les préoccupations d'ordre politique stimulent la volonté de promouvoir un développement régional, à travers les sociétés régionales d'investissements et la planification en milieu rural. Cette confirmation de l'aménagement a cheminé lentement dans les interstices de la planification économique.

\section{Difficultés de la décentralisation, fuite en avant néolibérale et désengagement de l'Etat (depuis 1990)}

9 A partir de 1987, de grands changements ont touché le pouvoir. Le nouveau gouvernement a opté pour une politique franche d'ouverture économique et d'intégration mondiale. Les objectifs majeurs de la décentralisation, devenue un discours dominant, portent sur un développement régional équilibré et sur une mise en valeur des régions peu peuplées de l'intérieur. Cependant, la décentralisation demeure un processus très encadré. La dépendance des collectivités locales vis-à-vis de l'administration centrale n'est pas seulement juridique, elle est aussi financière. La tutelle est d'autant plus lourde que les programmes de développement local doivent être conformes avec le plan national. Au Maroc, autre pays du Maghreb, le processus de décentralisation a pris, à partir de 2002, une nouvelle dimension avec la révision complète du régime juridique des collectivités locales pour réduire la tutelle de l'Etat. Le caractère $\mathrm{du}$ système politique tunisien privilégie en revanche une déconcentration très partielle plutôt qu'une véritable décentralisation. Selon Skander Ben Mami (2008), "cette déconcentration a apporté seulement des aménagements cosmétiques à la centralisation ». D'ailleurs, la médiocrité des résultats obtenus a amené les responsables à une réflexion sur l'aménagement du territoire basée sur des investissements économiques via l'équipement et la promotion administrative des villes moyennes. L'objectif était de remédier à la faiblesse de ces villes, tout d'abord directement, à travers le maillage et l'encadrement administratif et indirectement, à travers l'appareil productif touristique et industriel, ainsi qu'avec la décentralisation universitaire. La centralisation persistante du tertiaire de commandement à Tunis n'a pas favorisé le développement industriel régional.

Cependant, ces processus de déconcentration et de décentralisation ont posé le problème des échelons de la gouvernance, à plusieurs niveaux (communes, lieux informels ${ }^{9}$, intercommunalités). La croissance des flux des ruraux a alimenté la prolifération de l'habitat spontané autour des grandes villes et les structures de production déjà faibles des villes intérieures se sont détériorées (Chabbi, 1998). Cette situation a poussé l'Etat à abandonner progressivement son rôle de créateur d'emplois pour entamer d'autres actions dans des secteurs stratégiques (formations universitaires, nouvelles technologies d'information, etc.). Ces changements se sont répercutés sur la politique d'aménagement du territoire. L'espace se trouve toujours inégalement métropolisé par les services publics de niveau régional et les disparités persistent entre milieux urbain et rural. Le fait que certains programmes sociaux comme le FOPRODI, le FONAPRA, le SIVP ou le FIAP ${ }^{10}$ se soient à leur tour très concentrés sur le littoral explique aussi l'échec relatif des efforts réalisés.

11 L'adoption du Plan d'ajustement structurel en 1986, l'adhésion au GATT en 1990, les accords avec l'OMC en 1994 et la création d'une Zone de Libre-échange avec l'Union Européenne en 1996 ont entériné l'entrée de la Tunisie dans un processus d'ouverture 
aux marchés mondiaux impliquant un démantèlement du dispositif de production étatique, une stimulation de la compétitivité des entreprises tunisiennes et une privatisation des entreprises publiques. Cette nouvelle réalité a rendu nécessaire l'amélioration de la compétitivité des territoires, d'où la refonte en 1994 du code de l'aménagement du territoire et de l'urbanisme et l'élaboration de schémas directeurs d'aménagement dans les grandes agglomérations urbaines et les zones sensibles. Ces transformations politiques et institutionnelles liées au développement de l'aide internationale ont introduit également des changements significatifs de l'architecture administrative (Miossec, 2002). Le décret du 24 mars 1989 a établi une longue liste des compétences déléguées au gouverneur. Les communes doivent attendre la loi organique du 24 juillet 1995 pour connaître une évolution timide de leurs fonctions : l'urbanisme réglementaire est "communalisé »; le débat sur l'intercommunalité est lancé ; le fonctionnement des conseils municipaux est relativement amélioré. En effet, durant cette période, les communes ont été chargées de l'élaboration des plans d'aménagement urbains. Mais du fait de nombreuses distorsions, l'Etat fut contraint de mettre un terme en 2005 à cette expérience.

En outre, plusieurs agences spécialisées (AUGT, APAL) ou directions ministérielles sectorielles (patrimoine), ont vocation à établir des plans d'aménagement. Le District de Tunis, créé en 1975 pour réaliser les études et assurer la planification et la gestion du développement urbain du Grand Tunis, a été remplacé en 1994 après quelques tâtonnements par une agence urbaine chargée d'assister du point de vue technique les communes du Grand Tunis. La politique d'aménagement du territoire continu de connaitre des balbutiements. La séparation entre deux ministères de l'aménagement du territoire et de l'urbanisme, de 1994 jusqu'à 2003, en dit long à ce sujet ${ }^{11}$. Ces chevauchements de compétences entre administrations ont favorisé des conflits ${ }^{12}$.

En adoptant une logique fondée sur les idées d'efficacité et de compétitivité des villes, la stratégie tunisienne des années 1990 préconise une logique de métropolisation favorisant encore une fois les grandes villes côtières. Le deuxième schéma directeur d'aménagement du territoire national, réalisé en 1997, qui a été conçu en estimant que «la question est de refondre entièrement la conception de l'espace et par voie de conséquence de son aménagement", annonce la fin de la période où la pensée territoriale était construite autour du concept d'équilibre régional (Ben Letaief, 2008). SDATN tend ainsi à renforcer la structure spatiale de la Tunisie autour du pivot tunisois et de l'axe littoral oriental. Ce qui laisse penser que la recomposition de l'espace tunisien est envisagée en réponse à l'ouverture mondiale (Miossec, 2002).

14 Face aux risques associés au creusement des déséquilibres territoriaux, les pouvoirs publics tentent de rapprocher davantage l'administration du citoyen et de stimuler l'effort de développement des régions ${ }^{13}$. L'affinage progressif du maillage territorial a connu une nouvelle vague de promotion administrative des agglomérations. Le nombre de communes est passé de 212 en 1988 à 264 en 2008 et celui des secteurs (imadas) de 1749 à 2074. En conséquence, le nombre d'habitants des périmètres communaux a bondi de 4.477 .000 en 1988 à 6.746 .000 en 2008, soit deux tiers de la population du pays. Depuis, le moteur de la croissance urbaine est devenu de plus en plus endogène, alimenté par des redistributions internes et des migrations interurbaines, alors que l'exode rural se tarit. 


\section{Les jeux d'acteurs et les grands chantiers actuels sur fond de mondialisation}

\section{Un système territorial en pleine mutation}

15 L'intégration croissante de l'économie tunisienne aux échanges internationaux depuis les années 1990 a engendré de nouveaux défis : recomposition spatiale et sectorielle de l'activité économique, cohérence des politiques, protection de l'environnement, etc.La mondialisation actuelle se traduit par l'émergence de nouvelles commandes, de nouveaux acteurs et de nouvelles pratiques d'aménagement et d'urbanisme. Le désengagement de l'Etat, à travers la liquidation du secteur public de production des biens et des services, est l'arrière-plan de ce nouveau contexte.

Les institutions internationales s'imposent de plus en plus comme des acteurs de la commande spatiale et contribuent à une redéfinition des normes et des outils de l'aménagement à travers des directives (questions environnementales, développement durable, bonne gouvernance) (PNUD, 1999). La nouvelle politique d'aménagement du territoire semble entériner cette situation. Les documents officiels et les schémas d'aménagement actuels, notamment les nouveaux schémas directeurs d'aménagement $\mathrm{du}$ territoire et l'étude relative à la politique de la ville établie par le Ministère de l'Equipement et de l'Habitat ainsi que le $\mathrm{XI}^{\mathrm{e}}$ plan de développement économique et social (2007-2011), semblent opter pour des choix renforçant les tendances de développement sélectif à travers une métropolisation de plus en plus forte. Ceci a aggravé la ségrégation territoriale. En effet, la démarche de privatisation de la gestion des biens publics a connu, ces dernières années, une forte expansion ${ }^{14}$ via la concession d'un réseau GSM à un groupe égyptien en 2004, l'aménagement du Lac Sud de Tunis en 2007 (pour plus de 25 milliards de dollars) par le groupe émirati Sama Dubaï, l'aménagement du Lac de l'Ariana au Nord de Tunis par le groupe EL Maabar, ou encore en 2008, un projet de ville sportive et résidentielle baptisée Tunis sport city attribué au groupe Abu Khater. On peut ajouter à cette liste la construction d'un pôle financier confiée à un autre groupe émirati, ainsi qu'une raffinerie de pétrole à la Skhira, au sud de Sfax.Dans ce contexte, l'impératif d'efficacité « tend à supplanter le dogme du développement équilibré du territoire » (BenLetaief, 2008).

17 En réalité, face à une situation budgétaire difficile et sous prétexte que certaines fractions $\mathrm{du}$ territoire peuvent offrir une attractivité satisfaisante, les projets d'aménagement touchent inégalement les agglomérations. Dans ces conditions, un rééquilibrage est impossible. La tendance à la concentration des pouvoirs économiques, politico-administratifs et culturels dans les grandes villes du littoral se confirme. 
Illustration 2 - Cinquante ans d'aménagement du territoire entre réduction des déséquilibres et littoralisation

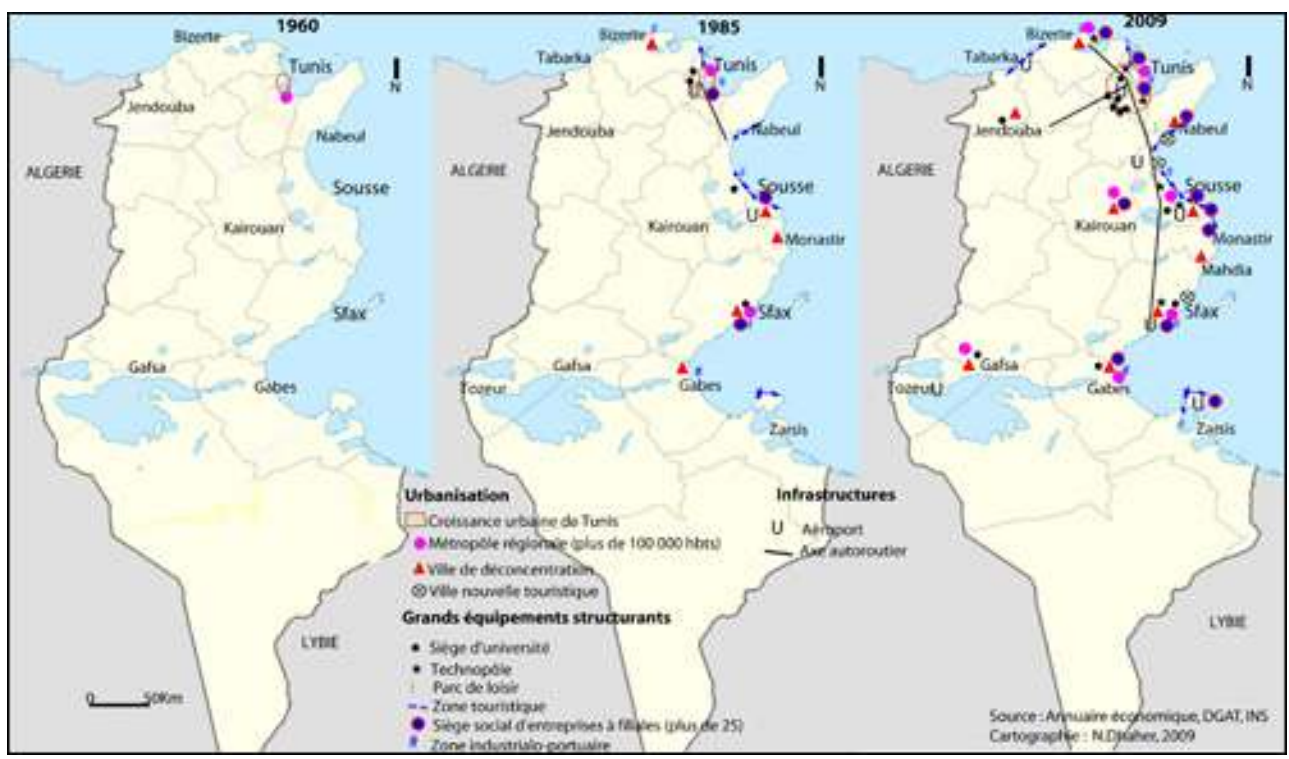

Source : annuaire économique, DGAT, INS

Ainsi organisé, l'espace géographique de la Tunisie sert-il aujourd'hui à améliorer le bien-être du citoyen tunisien, ou bien est-il simplement voué à satisfaire les demandes des investisseurs privés, nationaux ou extérieurs? L'orientation du dernier schéma national d'aménagement illustre le difficile compromis entre ces deux logiques (MEAT, 2002). Le document souligne d'une part que "l'intérêt national exigera que l'on mette l'accent sur les lieux les mieux situés pour faire face à la concurrence internationale ». Il ajoute dans le même temps "qu'il faudra veiller à ce que les différenciations économiques ne se transforment pas en distorsions sociales qui deviendraient insupportables ». Le schéma fixe désormais parmi les choix dont la mise en œuvre est prioritaire « la maîtrise de la fixité des habitants des régions de l'Ouest et des régions frontalières». D'autre part, "il ne faudra pas seulement répartir, mais aussi hiérarchiser et faire des choix ", écrivent les auteurs du SNAT.

\section{Gouvernance territoriale et développement durable : limites et opportunités}

La recomposition de l'espace urbain et la déstructuration d'une grande partie de l'espace rural sont plus que jamais un fait marquant. Les paysages urbains et ruraux affichent des contrastes et des paradoxes de plus en plus prononcés au niveau des infrastructures, des moyens de communication, de l'accès aux services de l'Etat et au niveau du rapport à la modernité. Certaines villes ont des conditions de vie proches du milieu rural et cumulent de nombreuses insuffisances (pollution atmosphérique ou visuelle, délabrement des voies de circulation et des trottoirs, état du bâti, etc.). Les grandes villes offrent une mosaïque de morceaux urbains hétéroclites produits selon des logiques conjoncturelles souvent contradictoires (médinas, cités de recasement, quartiers coloniaux, modernes, zones d'habitat non réglementaire, etc.). Les grandes agglomérations, notamment Tunis, Sfax et Sousse, souffrent de l'importance de l'habitat illégal, qui atteint $50 \%$ du bâti à Tunis, et de l'asphyxie due à un urbanisme 
souvent peu conforme aux exigences de la circulation (transport collectif, motorisation).

Actuellement, la population urbaine représente près des deux tiers de la population totale, contre près d'un tiers en 1956, avec trois types de régions : une région presque entièrement urbanisée, le District de Tunis, des régions moyennement urbanisées, avec des taux proches de la moyenne nationale (le littoral), et des régions plutôt rurales avec des taux inférieurs à $45 \%$ (la Tunisie intérieure). Dans la région qui s'étend de Sousse jusqu'à Chatt Mariem et bientôt à Hergla, ainsi que dans la région de Nabeul, sur une bande allant de Korba à Hammamet Sud, le développement de l'urbanisme balnéaire, tel qu'il est pratiqué actuellement sous forme de zoning, fait « table rase » des paysages ruraux. Les politiques environnementales restent encore, dans leur grande majorité, sectorielles et indépendantes les unes des autres. D'après un responsable de l'APAL, « la surexploitation foncière des côtes dans l'urbanisation et à travers l'activité touristique a un impact très lourd sur l'écosystème maritime ». L'analyse de la performance environnementale de la Tunisie, qui a été conduite par la Banque Mondiale en 2003, a souligné notamment que les zones touristiques ne font pas l'objet de réglementation visant la protection du milieu. Certains acteurs nationaux profitent de cette situation. L'étalement spatial cause une pression grandissante sur les zones sensibles telles que les terres agricoles fertiles et les paysages littoraux. Les zones sahariennes avec un fragile équilibre économique et social, ne sont plus épargnées. L'installation touristique dans des villes sahariennes comme Tozeur, Douz ou Nefta y a entraîné des changements perceptibles dans les modes d'utilisation et d'appropriation de l'espace.

Illustration 3 - Découpage foncier en zone touristique dans la ville saharienne de Douz

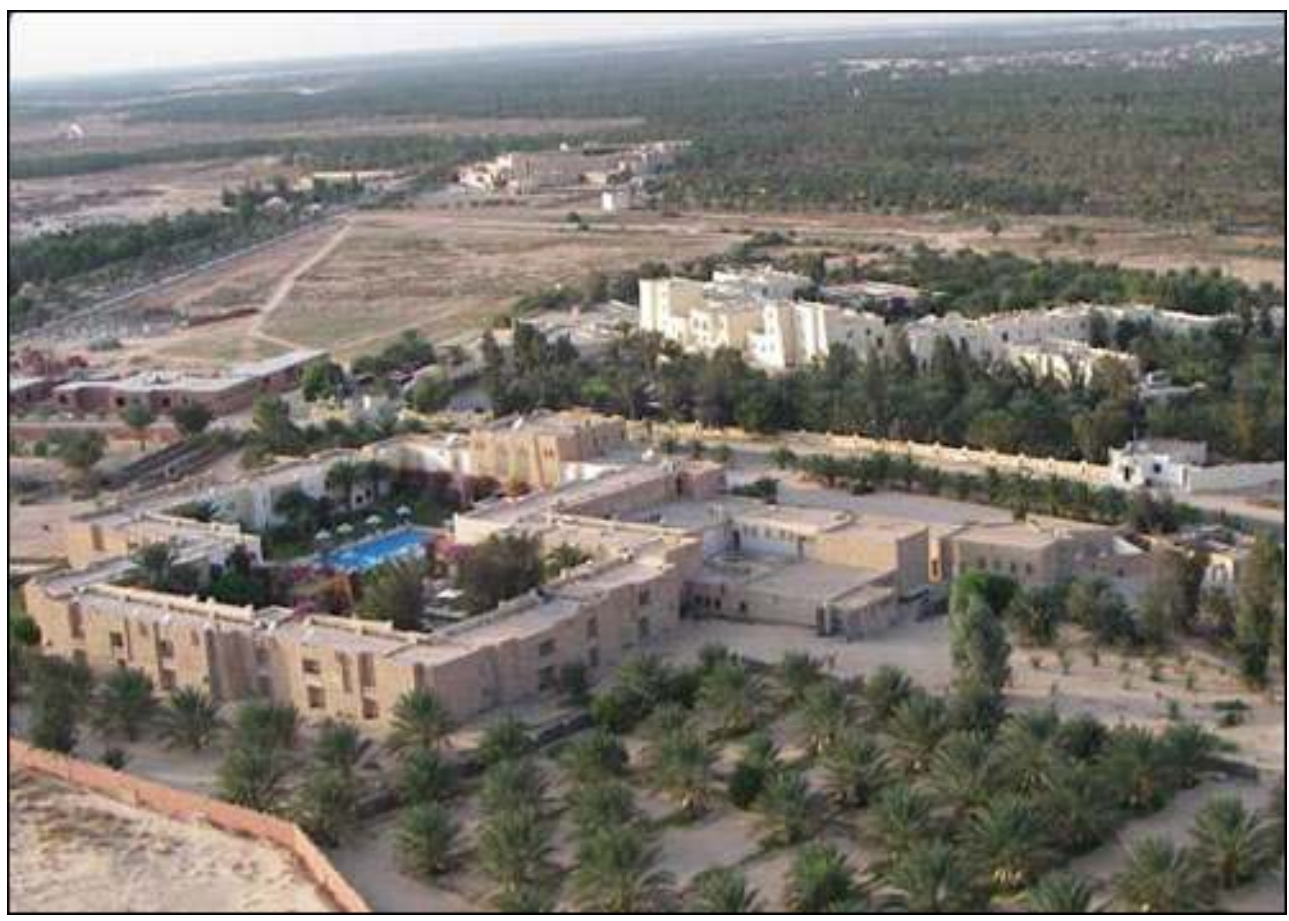

Source : archi-mag 2009.

21 La concentration soudaine d'équipements touristiques sur des espaces oasiens restreints a eu des conséquences multiformes (Picouet, 2002), notamment sur le plan environnemental. D'autre part, l'absence d'un marché foncier transparent favorise un 
étalement urbain informel et illégal dans et autour des grandes villes. Selon une étude d'URBACONSULT (2006), « ni les communes, ni les regroupements de communes, ni les agences foncières, ni les départements ministériels ne sont en mesure de constituer des réserves foncières pour satisfaire les besoins présents et les besoins futurs en logements, en équipements, en infrastructures et en zones d'activités ».

Ainsi, comme l'a déjà noté Ben Letaief (2008), « la démarche des pouvoirs publics reste hésitante et contradictoire, marquée par la persistance d'une logique centralisatrice et autoritaire d'une part, et la recherche forcée compte tenu de besoins de financement, de partenariat notamment avec le secteur privé ». Ce partenariat obéit souvent «à une logique de simple soulagement de l'Etat et de ses finances sans s'inscrire dans une réelle démarche démocratique de gestion participative ».

23 Toutefois, l'approche inédite lancée récemment dans une démarche volontariste d'élaboration de la stratégie de développement du Grand Sfax (projet Taparura de dépollution et d'aménagement du littoral nord de la ville de Sfax) s'est distinguée par l'enclenchement d'un processus participatif, mettant à contribution acteurs publics locaux, élus, professionnels, universitaires, ONG et citoyens.

\section{Illustration 4 - Le projet Taparura à Sfax}

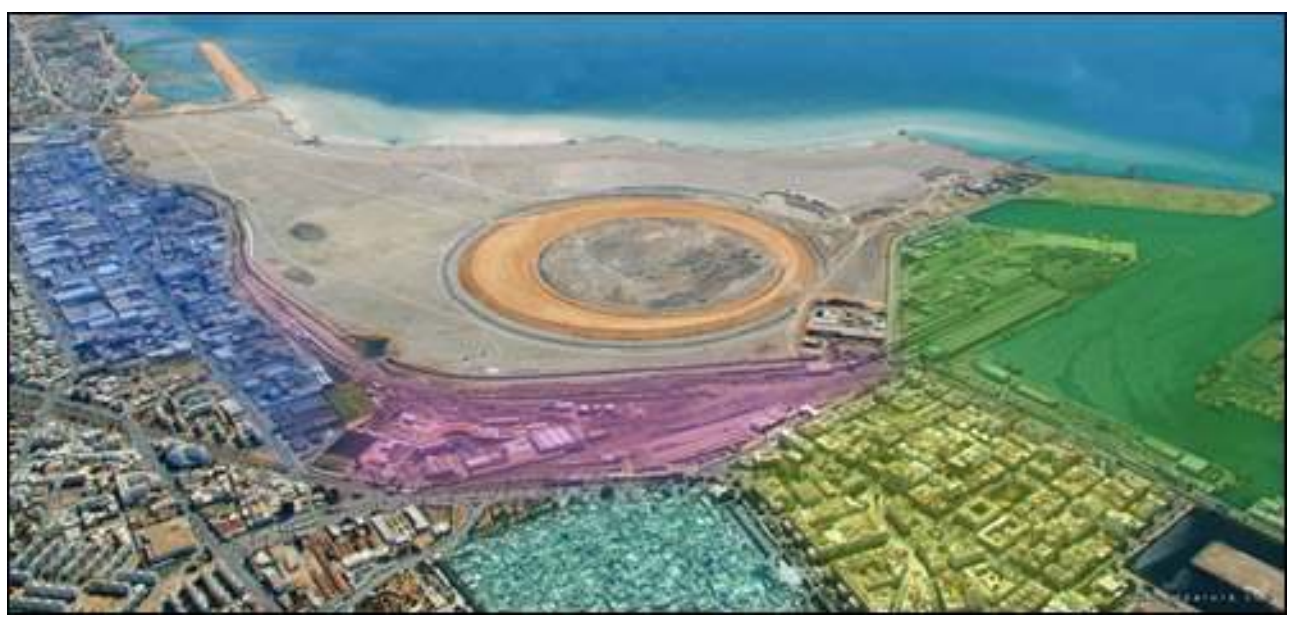

Source : DGAT 2010

A travers cette expérience, ne parait-il pas nécessaire de permettre aux différents acteurs locaux de définir les méthodes de gouvernance qu'ils estiment les plus appropriées dans une démarche bottom-up privilégiée pour l'émergence des projets? Au Maroc, où le concept de développement auto-centré semble dépassé, la récente réforme du paysage législatif a abouti à l'élaboration d'un schéma national d'aménagement du territoire (2005) basé sur la concertation, avec pour ambition de valoriser les spécificités territoriales, suivant la formule: " à chaque territoire selon ses potentialités économiques, à chaque territoire selon ses besoins sociaux ». En France, depuis les lois de décentralisation de 1982- 3, les décisions sur l'aménagement du territoire sont le résultat d'accords entre les collectivités territoriales, les régions et l'Etat, où les acteurs locaux ont une influence de premier plan. Reste à savoir comment de tels principes pourraient être promus dans un contexte comme celui de la Tunisie. 


\section{Conclusion}

Les politiques de développement et d'aménagement menées depuis l'Indépendance, souvent sans élaboration d'une doctrine d'ensemble, n'ont pas pu atténuer les disparités spatiales héritées de la colonisation et accentuées par l'ouverture à la mondialisation. Le nouvel ordre spatial, qui privilégie les lieux les mieux situés, les activités et les secteurs les plus portés vers l'extérieur, est en train d'accentuer les déséquilibres régionaux, tout en créant denouveaux problèmes environnementaux et territoriaux. La politique de métropolisation est devenue source d'iniquité et d'inefficacité (Davezies et Estèbe, 2007). Par ailleurs, le modèle centralisateur, bureaucratique et autoritaire en matière de gestion du territoire entretient la marginalité du local malgré les transformations des contextes internes et internationaux, qui tendent à délégitimer ces modes de gouvernement.

Enfin, l'option libérale, dans laquelle s'est engagé le régime tunisien à partir de 1986, ne semble pas être en mesure de sortir la Tunisie de l'impasse du sous-développement, du fait notamment du déficit de gouvernance, qui affaiblit la fourniture de biens publics. $\mathrm{Au}$ contraire, le néo-libéralisme, qui ne signifie « en rien [le] retrait de l'Etat et [la] fin de l'interventionnisme, mais [un] redéploiement des modalités d'exercice du pouvoir » (Hibou, 2006), éprouve le territoire tunisien sans en favoriser le développement.

A l'inverse, une politique de développement basée sur des projets locaux de coopération décentralisée et des stratégies à petite échelle, inspirée de nouveaux paradigmes comme ceux du développement durable ou de la gouvernance territoriale, pourrait ouvrir de nouvelles perspectives de développement aux territoires en Tunisie. Des processus de gouvernance territoriale - c'est-à-dire de concertation entre tous les acteurs du territoire - méritent de prendre le dessus sur les diktats des aménagistes ou des seules forces du marché.

\section{BIBLIOGRAPHIE}

Barthel P-A.,2003. Les lacs de Tunis en projets, reflets d'un nouveau gouvernement urbain. Annales de Géographie, volume 112, n 633, p. 518-536.

Belhédi A., 1992. Société, espace et développement en Tunisie. Tunis, PUT, FSHS, 262 p.

Belhadj A., 1990. Espace et société en Tunisie. Développement, organisation et aménagement de l'espace en Tunisie depuis l'indépendance. Revue tunisienne de géographie, $\mathrm{n}^{\circ}$ 18, p. 9-54.

Ben Letaief M., 2008. Les politiques urbaines en Tunisie : quelques réflexions sur les mutations d'une action publique postkeynésienne. Métropoles, $\mathrm{n}^{\circ} 4$.

Ben Letaief M., 1999. Le secteur public et la performance. Revue tunisienne de droit, numéro annuel, p. 133-177.

Ben Mami S., 2008. La décentralisation et la déconcentration en Tunisie et au Maroc, l'évolution du rôle des collectivités locales, des textes aux pratiques. IRG. 
Ben Mami S., Drossler L., Elie M., Gaouane Z., Gavrilov E., Meyer C., Widmeret S., 2007. Regards croisés sur la démocratisation et la gouvernance au Maghreb. Dossier, IRG.

Bethemont J., 2001. Géographie de la Méditerranée. A. Colin.

Bugnicourt J., 1971. Disparités régionales et aménagement du territoire en Afrique. A. Colin, 352 p.

Chabbi M., 1998. L'accès des pauvres au sol urbain en Tunisie - Tunis. Programme de gestion urbaine Tunisie.

Davezies L, Estèbe P., 2007. Mythes et légendes du développement territorial. L'autonomie politique dans l'interdépendance économique ? Pouvoirs Locaux, $\mathrm{n}^{\circ} 72$.

Hibou B., 2006. Le libéralisme réformiste, ou comment perpétuer l'étatisme tunisien. L'économie politique, $\mathrm{n}^{\circ} 32,112 \mathrm{p}$.

INS, Institut National de la Statistique, Recensements de la population et de l'habitat : 1966, 1984, 1994, 2004, Tunis.

Miossec J-M., 2002. Tunisie, métropolisation, mondialisation : efficience renforcée de l'axe oriental. Cahiers de la Méditerranée, volume 64, p.143-191.

Miossec J-M., Signoles P., 1984. Les Politiques urbaines en Tunisie, in Politiques urbaines dans le Monde Arabe, Métral et Mutin (dir), p. 183-202.

MEAT., Ministère de l'Environnement et de l'Aménagement du Territoire, Direction Générale de l'Aménagement du Territoire, 2002. Schéma directeur d'aménagement du territoire national, synthèse, Tunis.

PNUD., 1999. Rapport national sur le développement humain, République Tunisienne.

Picouet M., 2002. Formes de mobilité et dynamique de l'environnement en Tunisie. Revue Européenne des Migrations Internationales, volume 18, $\mathrm{n}^{\circ}$ 2, p. 51-65.

Signoles P., 1985. L'espace tunisien: capitale et Etat-Région. Tours, Urbama, 2 vol., 1041 p.

Urbaconsult., 2006. Evaluation et développement des instruments de l'aménagement du territoire en Tunisie. Rapport d'étude, DGAT, 114 p.

\section{NOTES}

1. Dans une situation difficile aux plans politique, économique et social, le premier président du pays, H. Bourguiba, a été évincé du pouvoir et remplacé par le président actuel Ben Ali.

2. L'aide a contribué à renforcer les cadres politiques et les fondamentaux institutionnels du pays, comme l'ont montré les réformes entreprises dans les années 1990.

3. Le pays était subdivisé en 70 caïdats, chaque caïdat correspond à un territoire tribal. Le Protectorat français a gardé cette structure tout en réduisant le nombre de caïdats à 36 après un processus de sédentarisation, parfois forcée, au sol, (Belhédi, 1990).

4. Le collectivisme est une orientation socialisante adoptée dans les années 1960, avec une vision relative à l'aménagement qui était réduite aux considérations économiques. Elle s'est soldée par un échec, d'où l'engagement vers le libéralisme économique.

5. Texte relatif à l'aménagement urbain (décret du 10/09/1943).

6. AFH (Agence Foncière de l'Habitat). ARRU (Agence de Rénovation et de Réhabilitation Urbaine). AUGT (Agence Urbaine du Grand Tunis). APAL (Agence de Protection et d'Aménagement du Littoral). PDR (Programme de Développement Rural). 
7. Plusieurs documents juridiques, techniques et institutionnels ont été mis en place durant la décennie 1970-1980 : Plan national d'aménagement du territoire (1970), Agences foncières (1973), Code de l'urbanisme (1979).

8. Dans les années 1980, devant les difficultés du contrôle des frontières, on créa trois nouveaux gouvernorats dans le Sud : Kébili, Tozeur et Tataouine qui sont, en fait, des zones frontalières détachées de leurs anciens gouvernorats (Gabès, Gafsa et Médenine).

9. Le secteur informel est toléré, il recouvre surtout l'installation des marchands " informels » dans toutes les villes, l'institutionnalisation des « souks libyens », etc.

10. FOPRODI (Fonds de la Promotion de la Décentralisation Industrielle). FONAPRA (Fonds National pour la Promotion de l'Artisanat et des Petits Métiers). SIVP (Stage d'Initiation à la Vie Professionnelle). FIAP (Fonds d'Insertion et d'Adaptation Professionnelle).

11. Le ministère de l'Environnement et de l'Aménagement du territoire, s'occupait de la planification régionale et territoriale alors que la planification intra-urbaine revenait au ministère de l'Equipement et de l'Habitat.

12. Comme par exemple lors de l'aménagement de la sebkha de l'Ariana au nord de Tunis (Barthel, 2003).

13. Le réseau routier a connu une évolution notable durant les vingt dernières années : la longueur totale des autoroutes est passée de $51 \mathrm{~km}$ en 1987 à 359,3 km en 2009. Les routes ayant une largeur égale ou supérieure à $7 \mathrm{~km}$ sont passées de $2082 \mathrm{~km}$ à $8662 \mathrm{~km}$.

14. Le gouvernement tunisien a cédé 217 entreprises publiques ou semi-publiques depuis le lancement du programme de privatisation en 1990.

\section{RÉSUMÉS}

$\mathrm{Au}$ lendemain de l'indépendance de la Tunisie, toute l'attention est accordée au développement économique et social et à la correction des grands déséquilibres régionaux hérités de la période coloniale. L'aménagement du territoire, thème mineur, amalgamé à la construction et à l'habitat, n'a émergé comme préoccupation majeure que lorsque le tourisme, retenu comme secteur de développement économique (1970), a engendré une démarche de planification spatiale. Depuis, l'Etat tunisien s'est donné plusieurs objectifs en matière d'aménagement du territoire, selon une démarche très centralisée. Le dispositif de planification spatiale qui a basculé d'une doctrine à l'autre, du socialisme à l'économie de marché, du collectivisme au néolibéralisme, a imprimé à l'espace, sous l'effet de différents facteurs historiques et socio-économiques, un développement inégal. Les mécanismes de la décentralisation et de la déconcentration n'ont pas modifié en profondeur les modalités d'intervention de l'Etat et n'ont pas contribué à maitriser les inégalités. Lanouvelle donne de la mondialisation a marqué une évolution économique et sociale en Tunisie. Aujourd'hui, on assiste à un glissement de référentiel et de légitimité des dogmes de l'équilibre spatial et du développement régional recherchés après l'indépendance du pays, vers celui de la compétitivité des villes et des régions. On se retrouve en présence d'une recomposition du territoire obéissant à des logiques nouvelles, celles du libéralisme et de la mondialisation. Le contraste spatial entre le littoral oriental et le reste du territoire s'en trouve renforcé, sur fond d'une logique autoritaire de gestion du territoire.

After Tunisia became independent, there was a focus on the economic and social development, and the project to remove the effects of the regional imbalance inherited from the colonial 
period. Town and country planning, first seen as minor topics, were linked to the building industry and housing. It is only when tourism became prominent in the 1970's with its corollary, spatial planning, that town and country planning were given attention. Since then, the Tunisian state set several goals in the fields of town and country planning, according to a very centralized approach. Spatial planning was conditioned by different doctrines, ie, socialism (a market economy), colonialism, neoliberalism, and by various historic and socioeconomic factors. But as a result, the development was uneven. By reason of decentralized mechanisms and the devolution, the modalities of the state intervention did not change deeply and did not contribute to overcome the disparities.

Globalization has brought about an economic and social evolution in Tunisia. Nowadays, we are witnessing the shift from the spatial balance and the regional development, which were inherent to the independence of the country, to the competitiveness of the cities and the regions. We are facing the reorganization of the country, with a new logics, that of liberalism and globalization. It is in a context of an authoritarian logic of management of the territory, that we put the emphasis on the spatial contrast between the Eastern coast and the rest of the country.

\section{INDEX}

Keywords : competitiveness, decentralization, disparities, globalization, governance, privatization, town and country planning, Tunisia

Mots-clés : aménagement du territoire, compétitivité, décentralisation, disparités, gouvernance, mondialisation, privatisation, Tunisie

\section{AUTEUR}

\section{NAJEM DHAHER}

Najem Dhaher est docteur en urbanisme et aménagement et Maître-assistant à l'Institut

Supérieur des Etudes Appliquées en Humanités de Tunis. najem_dhaher@yahoo.fr 